\title{
Etiology and Short term Outcome of Acute Kidney Injury (AKI) in Hospitalized Patients : A Single Center Study
}

\author{
Tania Mahbub ${ }^{*}$, Chowdhury Rifat Niger ${ }^{2}$, Rawshan Arra Khanam³ ${ }^{3}$ Mohammad Omar Faruq ${ }^{4}$
}

\begin{abstract}
:
Acute Kidney Injury (AKI) is a common cause of morbidity and mortality worldwide. However it is a treatable condition. Any disease when associated with AKI, mortality and morbidity increase manifolds. ${ }^{l}$ Early diagnosis and treatment is important to reduce morbidity, mortality and ICU admission. Causes of AKI may vary in country to country even within the country. International society of Nephrology has set a high ambitious goal "0 by 25" targeting zero death due to untreated AKI by 2025. Heading this challenge, it is important to know the causes of AKI worldwide. However, most of the AKI studies have been done in the developed countries and limited information is available regarding Asia, Africa and south America. Inspired by this slogan, this study was directed to find out the causes of AKI in admitted patients in United Hospital Limited between July 2015 to February 2017. Total 98 patients were included in the study. In our study, Infection and cardio-renal syndrome were the leading causes of AKI. Drug induced AKI also contributed a significant portion.
\end{abstract}

Key word: Acute kidney injury (AKI), Infection, Outcome.

\section{Introduction:}

Acute Kidney Injury (AKI) is the syndrome arising from a rapid fall in Glomerular Filtration Rate (GFR) over hours to days, characterized by retention of both nitrogenous and non-nitrogenous waste products of metabolism, as well as disordered electrolytes, acid-base and fluid homeostasis. Subjects diagnosed as AKI have radiologically normal size kidneys with maintained cortico-medullary differentiation (CMD). Epidemiologically, it has been found that 1 in 5 adults and 1 in 3 children worldwide experience AKI during a hospital episode of care. ${ }^{1}$ AKI occurs in $7 \%$ of hospitalized patients, out of which $65 \%$ of patients are admitted in ICU. Out of them, 20\%-25\% present with Sepsis \& $>50 \%$ present with Septic Shock. In ICU setting, mortality can be as high as $43 \%$ to $88 \%{ }^{2}$.

The epidemiology of AKI varies among countries and even within the same country. AKI is a leading cause of admission in United Hospital Limited as such our study was directed to find out the causes and short term outcome of AKI in our setting.

\section{Method:}

This observational cross sectional study was conducted at Department of Nephrology, United Hospital from July 2015

1. Associate Consultant, Dept. of Nephrology, United Hospital Ltd, Gulshan 2, Dhaka.

2. Specialist, Dept. of Gastroenterology, United Hospital Ltd, Gulshan 2, Dhaka.

3. Consultant, Dept. of Respiratory Medicine, United Hospital Ltd, Gulshan 2, Dhaka

4. Professor of Critical care Medicine \& Chief consultant, GICU \& Emergency, United Hospital Ltd, Dhaka

\section{*Corresponding Author:}

Dr. Tania Mahbub

Associate Consultant, Dept. of Nephrology,

United Hospital Ltd, Gulshan 2, Dhaka.

Email: taniaimran1@yahoo.com to February 2017, based on the research question: 'What are the causes of AKI in admitted patient \& what is the short term outcome?'

Primary objective was to Identify number and causes of AKI in hospitalized patients and secondary objective to see the outcome of AKI in terms of recovery, dialysis dependency and death. Also to get the percentage received renal replacement therapy (RRT) and intensive care unit (ICU) or coronary care unit (CCU) support.

We included all patients of AKI hospitalized in the dept. of nephrology, admitted \& treated under other care, visited by nephrologist. The exclusion criteria were patients with Chronic Kidney Disease (CKD), those who lost follow up, unwilling to participate in study.

The sample size was determined conveniently and it was, $\mathrm{n}=98$ patients. Convenient non probability sampling technique was used for collection of data for this study.

Depending on acute kidney injury network (AKIN) ${ }^{1}$ classification criteria, all patients were divided into three stages: stage 1, stage 2 and stage 3. Cardio-renal syndrome type 1 is characterized by acute worsening of cardiac function (pulmonary edema, cardiogenic shock, acute HF) leading to AKI. ${ }^{4}$ The study population was carefully observed for treatment outcome during the hospital stay in all three stages. Careful history alongside all clinical and bio-chemical parameters were recorded. End points were indicated by recovery (complete or partial), dialysis dependence or death during hospital stay. Data were analyzed by using SPSS version-16. Results are shown in percentages, tables, bars and charts depending on variables. Complete recovery means return of kidney function in terms of good urine output and normal blood biochemistry. Partial recovery means partial improvement of renal function.

Result:

98 patients were included as per selection criteria. Age range was from 19 years to 76 years \& male female ratio was $3: 2$. 
Diabetes, hypertension and ischemic heart disease were present in $24 \%, 20 \%$ and $14 \%$ study population respectively. Initial presentation is shown in Table 1. Figure 1 shows etiology of AKI. Infection was the leading cause of AKI in our study. Common causes of infection were Urinary Tract Infection (UTI), Pneumonia and septicemia. 15 patients had AKI due to UTI, 10 patients had Pneumonia and 7 patients had sepsis. Cardio-renal Syndrome was the next leading cause of AKI after infection. Total 25 patients developed AKI due to cardio-renal syndrome. The causes of AKI due to cardio-renal syndrome were cardiogenic shock, high ionotropic support, use of contrast and diuretics. 15 patients presented with drug induced AKI. The list of offending drugs is given in Table 2 . Five patients had AKI as a complication of pregnancy. Underlying causes of pregnancy induced AKI were toxemia of pregnancy, septic abortion and post-partum hemorrhage. Out of five patients of pregnancy induced AKI; one patient died and two patients were dialysis dependent at discharge. However, all the patients with pregnancy induced AKI had been transferred from inpatients of other hospitals. None of our pregnant OPD patients suffered from AKI requiring admission in hospital. AKI due to acute gastroenteritis (AGE) was least observed cause in our center. Two patients had AKI due to pancreatitis. Out of three patients who were admitted with AKI from glomerulonephritis, one had IgA nephropathy and two patients presented like Rapidly Progressive Glomerulonephritis (RPGN), biopsy showed mesangio capillary glomerulonephiritis. All of them improved and were dialysis independent at discharge.

Most of the malignant patients who were admitted with AKI presented with mild to moderate renal impairment due to chemotherapy, dehydration and tumor lysis syndrome. However, none of them needed dialysis and improved by conservative treatment. Bilimbi fruit poisoning caused renal failure in two patients. Both the patients recovered by conservative treatment. Three patients had rhabdomyolysis, among them two patients had AKI due to road traffic accident and one patient had rabdomyolysis secondary to very high dose of statin and fenofibrate. Figure 2 shows the number of patients in different stages (1,2 and 3) of kidney injury and corresponding number of patients needing dialysis. In stage I, 8 patients needed dialysis out of 21 patients those developed AKI. Among stage 2, there were 39 patients and 12 of them needed dialysis. In stage 3, 34 patients required dialysis out of 36 patients.

Figure 3 shows the outcome during hospital stay. Total 39 (39.7\%) patients needed either ICU or CCU support out of 98 patients.

Table 1:

\begin{tabular}{lc}
\hline & Total Patients 98 \\
\hline Increase serum creatinine & $84(86 \%)$ \\
Fever and systemic illness & $50(52 \%)$ \\
Oliguria/anuria & $53(55 \%)$ \\
Odema /Anasarca & $35(36 \%)$ \\
HTN & $46(47 \%)$ \\
Pulmonary oedema & $22(23 \%)$ \\
Ureamic syndrome & $20(21 \%)$ \\
\hline
\end{tabular}

Table 2:

\begin{tabular}{ll}
\hline Offending medications & Total affected patient $\mathbf{1 5}$ \\
\hline Colistin- 1 & Methotrexate-1 \\
Vancomycin- 1 & Phenofibrates -1 \\
Amikacin- 2 & Allopurinol -1 \\
Ketorolac- 4 & Yaba -1 \\
Beclophane-2 & ACE -1 \\
\hline
\end{tabular}

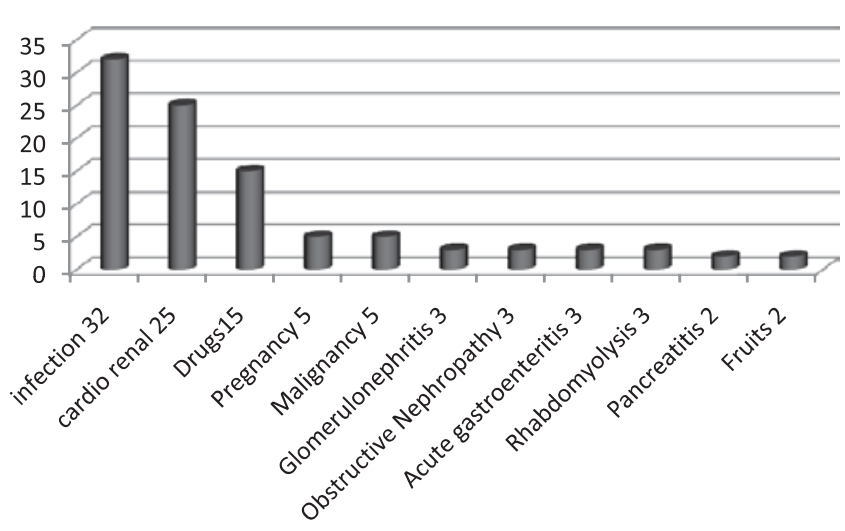

Figure 1

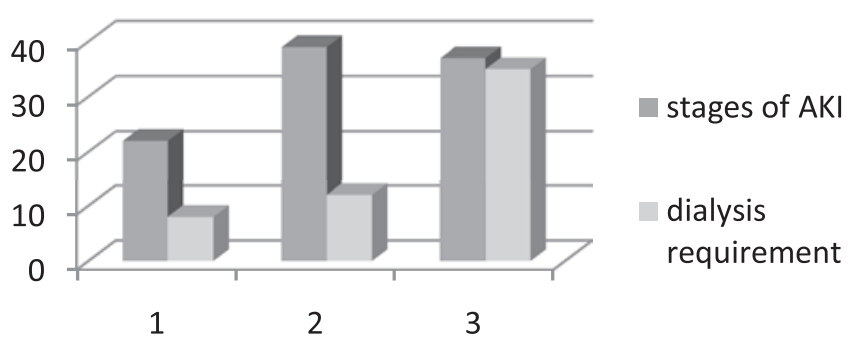

Figure 2

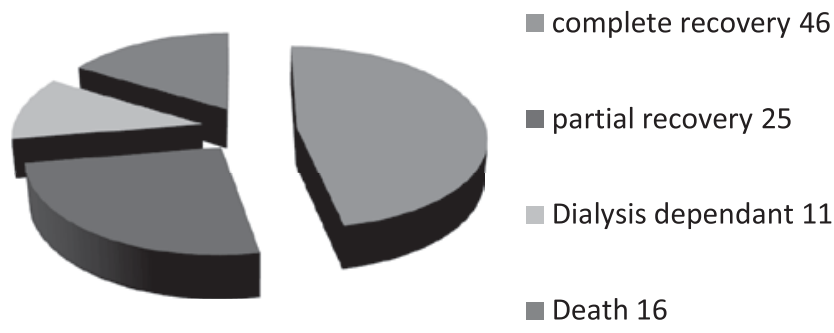

Figure 3

\section{Discussion:}

A meta analysis done by Susantitaphong et.a ${ }^{1}$ showed that the incidence rate of AKI is 1 in 5 adults $(21.6 \%)$ and 1 in 3 children $(33.7 \%)$ worldwide. There is very paucity of study on AKI in Bangladesh. According to Lopes et. al the common infections responsible for AKI were pulmonary (38.7\%), central nervous system (31.4\%), urological (9.2\%), abdominal origin (7.3\%) and endocarditis (4.1\%). Undetermined origin occurred in $9.3 \%$ of patients ${ }^{3}$. In our 
study, infection was the most common cause of AKI, 32 (32.6\%) and common causes of infection were UTI (15.3\%), pneumonia (10.2\%) and sepsis (7.1\%)

United hospital is one of the largest tertiary care cardiac centers of the country. It is not surprising that a number of patients develop AKI in course of their illness due to cardio renal syndrome. In our study, cardio renal syndrome had been the second most common cause of AKI after infection. Approximately $27 \%$ of patients admitted with acute decompensated heart failure developed AKI. ${ }^{4}$ Susantitaphong et.al ${ }^{1}$ also stated in their study that rate of AKI is high, $24.3 \%$ followed by cardiac intervention.

It has already been well established that analgesics are responsible for AKI. Use of nephrotoxic drugs was the third common cause in our study. NSAIDs and other nephrotoxic drugs were the main culprit. There were some other agents that caused nephrotoxicity like beclophene, high dose statin and fenofibrate and methotrexate. In one study ${ }^{5}$, aspirin and acetaminophen were used regularly by 37 percent and 25 percent patients, respectively. Among them renal failure developed in 19 percent and 12 percent patients respectively. Besides all common nephrotoxic medication, we had 2 patients suffering from AKI due to methotrexate toxicity. But in both cases they had taken methotrexate in higher dose mistakenly. One died due to multi organ failure from severe methotrexate toxicity.

In our study, the main cause of AKI in pregnancy were Septic abortion, Toxaemia of pregnancy and Post partum haemorrhage. However, all of the patients had been referred from other center and we did not have pregnancy induced AKI among those who were in regular follow up patients. Regular pre natal follow up may be one of the protective factors against development of AKI during pregnancy.

There are limited information regarding pregnancy induced AKI in Bangladesh. In India one study concluded that AKI is a common finding in pregnancy. According to this study the common causes of pregnancy related AKI were Sepsis (59\%), pre-eclampsia, and eclampsia (56\%). ${ }^{6}$ Huang $\mathrm{C}$ et al. reported $0.81 \%$ incidence of AKI during pregnancy with about $17 \%$ women with pre-eclampsia/eclampsia and $60 \%$ women with HELLP syndrome developing AKI. ${ }^{7}$ Another study done in Canada over a period of 15 years concluded that $\mathrm{AKI}$ is a rare complication of pregnancy. They conducted their study over 1.9 million pregnants and stated only 1 in 10,000 pregnancies needed dialysis. The causes in that study were Toxaemia of pregnancy and Sepsis. ${ }^{8}$

AKI is one of the most common complications in patients with Severe Acute Pancreatitis (SAP). We had only 3 cases of AKI due to pancreatitis. One study showed $35.8 \%$ patients with SAP may develop AKI. ${ }^{8}$ In another study, out of 554 patients with pancreatitis 24 (4.4\%) developed AKI. ${ }^{10}$

In our study, AGE had been the least observed cause. Only 5 patients had AKI from AGE. This may be due to good response of patients to initial fluid therapy. According to one study from India, AKI due to gastroenteritis in developing countries is not uncommon. In that study out of 100 patients
$96 \%$ patients recovered and $4 \%$ died. This study claims that AGE is the 3 rd most common cause of AKI in elderly patient. ${ }^{11}$

Glomerulonephritis (GN) may cause AKI. In our center GN had been the least observed. We had only 3 patients, who required hospital admission. The reason behind that most of the GN patients were treated as out patient basis and required admission only when there is an abrupt rise of serum creatinine or the presentation like RPGN or accompanied by severe renal impairment. In a study on glomerulonephritis conducted over 25 years, authors found GN comprises only $4.1 \%$ of total AKI. Among them $14.4 \%$ were Post infectious, Lupus nephritis comprised $8.5 \%$, Mesangiocapillary type was $3.4 \% .^{12}$

Two patients in our study were admitted due to bilimbi fruit (Averrhoa bilimbi) poisoning. A case report stated bilimbi can cause acute renal shut down when ingested in 150 to $200 \mathrm{~mL}$ of fruit juice in an empty stomach. This results in acute renal tubular blockage by calcium oxalate crystals. ${ }^{13}$

AKI associated worldwide mortality rate was reported to be $23.9 \%$ in adults. ${ }^{1}$. Lopes et. al reported the mortality rate to be $25.3 \% .^{3}$ In our study overall mortality was $16.3 \%$. Mortality is very high according to some study when pregnancy is associated with AKI. One study showed Maternal and fetal mortality were $20 \%$ and $22 \%$ respectively and sepsis was the leading cause of maternal mortality. ${ }^{6}$ Mortality rate may be as high as $58 \%$, when associated with pancreatistis. ${ }^{9}$ However, as we have a limited number of cases with pregnancy and pancreatitis associated AKI we are unable to make a conclusion on these findings.

\section{Conclusion:}

In our study infection was the leading cause of AKI. Cardiorenal Syndrome type 1 was the second most common cause of AKI indicating a need for aggressive measures. Caution should be taken while using nephrotoxic medications. AGE and pregnancy induced AKI are less common in our study. Pregnancy induced AKI is not commonly observed in our patients as they get regular prenatal follow up.

This study aimed only to assess the short term outcome of AKI during hospital stay and did not aim in studying the long term squeal of kidney injury. A multi-center study aiming to assess both short term and long term outcome of AKI will offer a more accurate picture of AKI in hospitalized patients in Bangladesh.

\section{References :}

1. Susantitaphong P, Cruz DN, Cerda J, Abulfaraj M, Alqahtani F;Acute Kidney Injury Advisory Group of the American Society of Nephrology. World incidence of AKI: a meta-analysis. Clin J Am Soc Nephrol. 2013 ;8(9):1482-93. doi: 10.2215/CJN.00710113.

2. Ricci Z, Ronco C, D'Amico G, De Felice R, Rossi S, Bolgan I et.al. Practice patterns in the management of acute renal failure in the critically ill patients: an international survey. Nephrol Dial Transplant. 2006;21(3):690-6.

3. Lopes JA, Jorge S, Resina C, Santos C, Pereira A, Neves J et al. Acute renal failure in patients with sepsis. Crit Care. 2007;11:411. 
Bangladesh Crit Care J September 2019; 7 (2): 77-80

4. Forman DE, Butler J, Wang Y, Abraham WT, O'Connor CM, Gottlieb SS et al. Incidence, predictors at admission, and impact of worsening renal function among patients hospitalized with heart failure. J Am Coll Cardiol 2004; 43: 61-67.

5. Fored CM, Ejerblad E, Lindblad P. Acetaminophen, aspirin, and chronic renal failure. N Engl J Med. 2001;345(25):1801-1808.

6. Mahesh E, Puri S, Varma V, Madhyastha P R, Bande S, Gurudev K C. Pregnancy-related acute kidney injury: An analysis of 165 cases. Indian J Nephrol.2017;27(2):113-7.

7. Huang C., Chen S. Acute kidney injury during pregnancy and puerperium: a retrospective study in a single center. BMC Nephrol. 2017;18(1):146.

8. AM, Liu K, Shariff SZ, Ray JG, Sontrop JM, Clark WF et al. Characteristics and outcomes of AKI treated with dialysis during pregnancy and the postpartum period. J Am Soc Nephrol. 2015;26(12):3085-3091
9. Compañy L, Sáez J, Martínez J. Factors predicting mortality in severe acute pancreatitis. Pancreatology 2003;3(2):144-8.

10. Ljutić D, Piplović-Vuković T, Raos V, Andrews P. Acute renal failure as a complication of acute pancreatitis. Renal Fail 1996;18(4):629-33.

11. Inbanathan J, Lavanya B U. Clinical Profile of Renal Involvement in Acute Gastroenteritis Patients. International Journal of Scientific Study.2016;4(8):48-52.

12. Naqvi R, Mubarak M, Ahmed E, Akhtar F, Bhatti S, Naqvi A et al.Spectrum of glomerular diseases causing acute kidney injury; 25 years experience from a single center. J Renal Inj Prev. 2015; 4(4): $113-116$.

13. Billah, M.M., Rahman, M.A., Rahim M.A., Swarna A., Mitra P.,Chowdhury T. et al. Acute Kidney Injury Following Ingestion of Averrhoa bilimbi Juice. Bangladesh Critical Care Journal.2015; 3(2): 71-73 